\title{
A Cross-sectional Study on Chronic Fungal Rhinosinusitis in a Tertiary Care Hospital in Central Delhi, India
}

\author{
Prafulla Songara*1 $^{(D)}$, Gaurav Saxena ${ }^{1}$ (D) and Ramesh Agrawal² \\ ${ }^{1}$ Department of Microbiology, Government Medical College, Ratlam, Madhya Pradesh, India. \\ ${ }^{2}$ Department of Microbiology, Government Medical College, Khandwa, Madhya Pradesh, India.
}

\begin{abstract}
Chronic Rhinosinusitis is a common disorder, and its prevalence vary from $1-20 \%$ globally. The incidence of fungal sinusitis has increased to such extent in recent years that fungal infection is a common diagnosis in patients with Chronic Rhinosinusitis. The objectives of this current research were objectives of estimating the prevalence of Fungal aetiology in chronic sinusitis patients and their drug sensitivity pattern with common antifungal drugs. A total of 61 Cases present with Chronic Rhinosinusitis (CRS), visited in a tertiary care hospital based in Central Delhi, were included in our study. Excision of sinus tissue, including polyps and masses, were collected in the operation theatre during Functional Endoscopic Sinus Surgery (FESS) procedure in a sterile manner. All the tissues brought in sterile normal saline were processed for bacteriological and mycological examination. Tissues, obtained in $10 \%$ formalin were processed for histopathological and cytological analysis. A total of 14 (22.9\%) cases of Chronic Rhinosinusitis were affected by fungal etiologies. By E test, the MIC range for isolates of Rhizopus arrhizus after $24 \mathrm{hr}$ of incubation was $1-2 \mu \mathrm{g} / \mathrm{mL}$, and the mean was $1.5 \mu \mathrm{g} / \mathrm{MI}$. Similarly, the MIC range for isolates of Aspergillus flavus after $48 \mathrm{hr}$ of incubation was $0.5-16 \mu \mathrm{g} / \mathrm{mL}$, and the mean was $4.09 \mu \mathrm{g} / \mathrm{mL}$. By the M38-A broth dilution method, the MIC range for the isolates of Rhizopus arrhizus after $24 \mathrm{hr}$ of incubation was $0.5-2 \mu \mathrm{g} / \mathrm{mL}$, and the mean was $1.25 \mu \mathrm{g} / \mathrm{ml}$. Similarly, the MIC range for isolates of Aspergillus flavus after $48 \mathrm{hr}$ of incubation was $0.5-4 \mu \mathrm{g} / \mathrm{mL}$, and the mean was $1.95 \mu \mathrm{g} / \mathrm{mL}$.
\end{abstract}

Keywords: Rhinosinusitis, New Delhi, Fungus, Aspergillus flavus, Rhizopus arrhizus, India

*Correspondence: drprafulla07@gmail.com

(Received: January 10, 2021; accepted: February 04, 2021)

Citation: Songara P, Saxena G, Agrawal R. A cross-sectional Study on Chronic Fungal Rhinosinusitis in a Tertiary Care Hospital in Central Delhi, India. J Pure Appl Microbiol. 2021;15(1):279-284. doi: 10.22207/JPAM.15.1.21

(C) The Author(s) 2021. Open Access. This article is distributed under the terms of the Creative Commons Attribution 4.0 International License which permits unrestricted use, sharing, distribution, and reproduction in any medium, provided you give appropriate credit to the original author(s) and the source, provide a link to the Creative Commons license, and indicate if changes were made. 


\section{INTRODUCTION}

Rhinosinusitis is a spectrum of inflammatory and infectious diseases that involve one or more of the paired paranasal sinuses and nasal mucosa. Because of the contiguous relationship of the sinuses and nasal mucosa, rhinitis and sinusitis coexist and are concurrent in most individuals ${ }^{1}$.

Chronic Rhinosinusitis (CRS) is a multifactorial illness. Air pollution, viruses, bacteria, fungi, genetic factors, immune deficiency, and anatomical abnormalities within the sinus system may play a contributory role in the same patient. It is often difficult to define a precise cause of illness in an individual patient ${ }^{2}$. Gender, geographical location, and race or ethnicity influence the frequency of disease ${ }^{3}$.

Chronic Rhinosinusitis is a common disorder, and its prevalence is $1-20 \%$ in different parts of the world ${ }^{4}$. CRS poses an immense economic burden to the patient via the treatment cost and the indirect costs due to restricted activity days ${ }^{5}$. Many patients with chronic sinus disease take multiple courses of antibiotics and surgeries with little or no improvement in their condition. Despite the tremendous advances in medicine over the last few decades, there have been relatively few advances in the diagnosis and treatment of chronic sinus disease $e^{6,7}$.

Previously fungal Rhinosinusitis was considered a uncommon disease, but it has gained importance in recent past due to increase in the incidence. The incidence of fungal sinusitis has increased to such extent in recent years that fungal infection is a common diagnosis in patients with Chronic Rhinosinusitis. Whether fungi can exist in the sinus mucous without causing disease is unclear. However, over the last two decades, there is an increased frequency of recognized and reported cases of fungal Rhinosinusitis worldwide ${ }^{8}$.

Due to increased cases of invasive fungal infection in immunocompromised patients and emergence of resistance to antifungal agents it became essential to perform drug susceptibility testing in mold infections $s^{8,9}$.

In our literature review, we have not found any study on the pattern of antifungal drug sensitivity among chronic fungal sinusitis cases in New Delhi. Therefore, we did this current research with the objectives of estimating the prevalence of Fungal aetiology in chronic sinusitis patients and their drug sensitivity pattern with common antifungal drugs.

\section{MATERIALS AND METHODS}

This cross-sectional study was conducted in the Departments of Microbiology, Pathology, and Otorhinolaryngology of Lady Hardinge Medical College (LHMC) and associated hospitals, New Delhi. Data collection was done in the year 2010. A total of 61 eligible cases, as per our inclusion and exclusion criteria, visited our hospital, were included in our study.

\section{Inclusion criteria}

1. Cases present with Chronic Rhinosinusitis (CRS) in Hospital OPD (inflammation of paranasal sinus for more than 12 weeks with or without radiological evidence).

2. Age of participant should be more than 14 years.

Every participant was provided with a patient information sheet and informed written consent was taken from each enrolled patient. Three such patients who has not given consent to participate in the study were excluded.

\section{Sample collection and transport}

Excised sinus tissue, including polyps and masses, were collected in the operation theatre during Functional Endoscopic Sinus Surgery (FESS) procedure in a sterile manner. The specimen was divided into two separate parts. One part was kept in a sterile container having sterile normal saline which is used for bacteriological and mycological examination. The other part was placed in $10 \%$ formalin, used for histopathological examination. These samples were transported and processed in Microbiology and Pathology laboratories immediately.

\section{Sample processing}

All the tissues brought in sterile normal saline were processed in the Microbiology department, LHMC for bacteriological and mycological examination. Tissues, obtained in $10 \%$ formalin were processed for histopathological and cytological examination in the department of Pathology LHMC, New Delhi.

\section{Antifungal drug susceptibility testing}

Antifungal drug susceptibility testing for Amphotericin B was performed for all the isolates using the CLSI M38-A broth dilution method and 
E-test method following the instructions of the manufacturer ${ }^{10}$.

\section{RESULTS}

A total of 64 eligible patients were contacted for the present study. Few (3) Patients refused to participate in the study, so the final sample size was 61 . In the current study, 34 (55.7\%) males and 27 (44.2\%) females were included. The most common (47.5\%) age group involved was 3150 years, followed by less than 30 years (31.1\%) and $51-70$ years $(21.3 \%)$.

On culture of samples from 61 patients of CRS, a total of 21 isolates were identified and out of which, 13 were fungal, and 8 were bacterial.

Out of 61 samples, seven samples were positive for $\mathrm{KOH}$, culture, and histopathology, six samples were positive for $\mathrm{KOH}$ and culture, and one was positive for $\mathrm{KOH}$ and histopathology. Therefore, a total of 14 (22.9\%) cases of Chronic Rhinosinusitis were affected by fungal etiologies. Out of these 14 cases fungal agent were isolated from 13 patients. Out of these 13 isolates 11 (84.61\%) were Aspergillus flavus and 2 (15.38\%) isolates were Rhizopus arrhizus [Table 1]

In this study, among all chronic fungal sinusitis patients, most ( 7 cases, $50.0 \%$ ) of the participants presented in summer season followed by winter season (5 cases, 35.7\%) and the rainy season (July to October, 2 cases, 14.2\%).

By $E$ test, the MIC range for isolates of Rhizopus arrhizus after $24 \mathrm{hr}$ of incubation was 1-2 $\mu \mathrm{g} / \mathrm{mL}$, and the mean was $1.5 \mu \mathrm{g} / \mathrm{Ml}$. Similarly, the MIC range for isolates of Aspergillus flavus after 48 $\mathrm{hr}$ of incubation was $0.5-16 \mu \mathrm{g} / \mathrm{mL}$, and the mean was $4.09 \mu \mathrm{g} / \mathrm{mL}$. [Table 2]

By the M38-A broth dilution method, the
MIC range for the isolates of Rhizopus arrhizus after $24 \mathrm{hr}$ of incubation was $0.5-2 \mu \mathrm{g} / \mathrm{mL}$, and the mean was $1.25 \mu \mathrm{g} / \mathrm{ml}$. Similarly, the MIC range for isolates of Aspergillus flavus after $48 \mathrm{hr}$ of incubation was $0.5-4 \mu \mathrm{g} / \mathrm{mL}$, and the mean was $1.95 \mu \mathrm{g} / \mathrm{mL}$. [Table 3]

On comparison, MICs results of E-test and CLSI standard broth dilution testing (M38-A) showed MICs of all isolates were within $\pm 1 \log 2$ dilution for $R$. arrhizus and agreement was found to be $100 \%$ for both $\pm 1 \log 2$ and $\pm 2 \log 2$ dilution. Similarly, MICs of most of the isolates of Aspergillus flavus showed $45.45 \%$ and $90.90 \%$ agreement at $\pm 1 \log 2$ and $\pm 2 \log 2$ dilution, respectively.

\section{DISCUSSION}

In a present study, a total of 61 samples from patients of chronic Rhinosinusitis were included. Out of them, 59 samples were polyps/ tissue/ mass collected from the patients who underwent functional endoscopic sinus surgery, and the remaining two samples were sinus secretions from patients managed conservatively. In our study, we observed that 34 (55.73\%) CRS patients were males, and 27 (44.27\%) were females. So, the prevalence of chronic Rhinosinusitis in male patients was 1.25 times higher than females. Aghakhani et al. and Razmpa et al. also observed similar findings among the CRS group, $58 \%$ male and $42 \%$ females, and $68 \%$ male and $32 \%$ female, respectively.

In our study, we observed that 34 (55.73\%) CRS patients were males, and 27 $(44.27 \%)$ were females. So, the prevalence of chronic Rhinosinusitis in male patients was 1.25 times higher than females. Aghakhani et al. ${ }^{11}$ and Razmpa et al. ${ }^{12}$ also observed similar findings

Table 1. Distribution of Chronic Fungal Rhino-sinusitis cases according to $\mathrm{KOH}$, culture, and Histopathology finding

\begin{tabular}{lcccl}
\hline $\begin{array}{l}10 \% \\
\mathrm{KOH}\end{array}$ & $\begin{array}{c}\text { Fungus on } \\
\text { Culture }\end{array}$ & $\begin{array}{c}\text { Fungal } \\
\text { Histopathology } \\
\text { (H/P) }\end{array}$ & Number & Cultured isolates \\
\hline Yes & Yes & Yes & 7 & $\begin{array}{l}13 \\
11(84.61 \%) \text { Aspergillus flavus and } \\
\end{array}$ \\
& & & $2(15.38 \%)$ Rhizopus arrhizus \\
Yes & Yes & No & 6 & 0 \\
Yes & No & Yes & 1 & 0 \\
No & No & No & 47 & 0 \\
Total & & 61 & 13 \\
\hline Journal of Pure and Applied Microbiology & & 281
\end{tabular}


Table 2. In vitro susceptibilities of fungal isolates to Amphotericin-B as determined by E-test

\begin{tabular}{lcc}
\hline \multirow{2}{*}{ Parameter } & \multicolumn{2}{c}{ Isolates } \\
\cline { 2 - 3 } & R. arrhizus & A. flavus \\
\hline Incubation time & $24 \mathrm{hr}$. & $48 \mathrm{hr}$. \\
MIC range $(\mu \mathrm{g} / \mathrm{ml})$ & $1-2$ & $0.5-16$ \\
Mean $(\mu \mathrm{g} / \mathrm{ml})$ & 1.5 & 4.09 \\
\hline
\end{tabular}

Table 3. In vitro susceptibilities of fungal isolates to Amphotericin B as determined by CLSI M38-A broth dilution method

\begin{tabular}{lcc}
\hline Parameter & \multicolumn{2}{c}{ Isolates } \\
\cline { 2 - 3 } & R. arrhizus & A. flavus \\
\hline Incubation time & $24 \mathrm{hr}$. & $48 \mathrm{hr}$. \\
MIC range $(\mu \mathrm{g} / \mathrm{ml})$ & $0.5-2$ & $0.5-4$ \\
Mean $(\mu \mathrm{g} / \mathrm{ml})$ & 1.25 & 1.95 \\
\hline
\end{tabular}

among the CRS group, $58 \%$ male and $42 \%$ females, and $68 \%$ male and $32 \%$ female, respectively.

In the present study majority (26.2\%) of CRS, patients were seen in $31-40$ years of age group, followed by $24.6 \%$ in $21-30$ years of age group. Aghakhani et al. ${ }^{11}$ found the majority of CRS patients (24\%) in $30-39$ years, followed by $20 \%$ in 40-49 years of age group.

FRS was diagnosed in age groups ranging from 17 to 63 years. Most cases were from 31-40 years of age group, and the mean age was 34.3 years. Study done by Razmpa et al. ${ }^{12}$ also found highest number of cases in 30-39 years of age group, with mean age of 37 years. Venugopal et al. ${ }^{13}$ found the age range was from 16 to 68 years (average: 35 years). Chakrabarti et al. ${ }^{14}$ in their study found that younger age groups are more prone to fungal Rhinosinusitis. Jahromi et al. ${ }^{15}$ reported age ranging from 17 to 58 years (mean of 33.4 years). As per our study no specific age group can be considered as risk factor for the development of FRS.

The FRS observed in the present study was $22.9 \%^{14}$, based on $\mathrm{KOH}$, culture, and histopathology among the patients with the clinical presentation of chronic Rhinosinusitis. Worldwide prevalence rate varies from $6.7 \%$ to $75.5 \%$. In comparison to other studies from various parts of India we observed less prevalence rate. Chakrabarti et al. ${ }^{14}$ from Chandigarh and Venugopal et al. ${ }^{13}$ from Tamil Nadu reported $42 \%$ and $45 \%$ of prevalence rate, respectively. Singh et al. ${ }^{16}$ identified fungal elements in $62.1 \%$ of surgical specimens from patients with Chronic Rhinosinusitis in Gujarat.

From outside India, in Brazil Dall'Igna et al. ${ }^{17}$ estimated $6.7 \%$ prevalence of fungal Rhinosinusitis. In Nepal, paranasal mycosis was found to be $14 \%$ in chronic maxillary sinusitis ${ }^{18}$. In Malaysia, the prevalence of allergic fungal Rhinosinusitis among patients with chronic refractory sinusitis was $26.7 \%{ }^{19}$. In an Iranian study, paranasal mycoses were proved in $46 \%$ of cases with suspected fungal sinusitis ${ }^{15}$. Another study from Iran found a $42 \%$ prevalence rate on direct microscopy with nasal polyposis ${ }^{12}$. Braun et al. ${ }^{20}$, in Europe, found $75.5 \%$ surgical specimens from patients with Chronic Rhinosinusitis with or without polyposis were positive for fungal elements.

Except for a few reports, our results fall in between most of the studies. By using improved and novel samples processing technique for the detection of fungi significantly higher prevalence rate has been reported in some studies. However, similar methods were adopted in one study still gave a low prevalence rate ${ }^{19}$. Different climate conditions in different geographical areas may be the reason for the varying in prevalence of pathogenesis of FRS.

In our study, out of 14 , only 8 (57\%) samples showed fungal elements in histopathology. Similarly, Aghakhani et al. ${ }^{11}$ found fungal culturepositive in 49 (49\%) of cases of paranasal sinuses and on histological examination fungal elements ware seen in 41 (41\%) of cases.

Aspergillus flavus which is isolated in $84.61 \%$ cases, was the most frequent causative fungi and this finding is concordant to other studies conducted in various part of world. Rao et al. ${ }^{21}$ isolated from $87.5 \%$ of Aspergillus flavus. Chakrabarti et al. ${ }^{14}$ identified the same species from $80 \%$ of cases. Similarly, Panda et al. ${ }^{22}$ isolated Aspergillus flavus from $79.7 \%$.

From outside India, Razmpa et al. ${ }^{12}$, and Jahromi et al. ${ }^{15}$ from Iran isolated Aspergillus flavus as a commonest spp. Rahman et al. ${ }^{23}$ from Saudi Arabia also separated similar spp.

In our study, the prevalence of fungal 
Rhinosinusitis in male patients was 1.3 times higher than females. Iwen et al. ${ }^{24}$ also found male: female ratio in fungal rhinosinusitis 1.125:1. Chakrabarti et al. ${ }^{14}$ also reported male predominance with male: female ratio being 2.8:1.

To know the antifungal sensitivity/ resistant pattern of the fungal isolates from chronic Rhinosinusitis, we tested all isolates for antifungal susceptibility by E-teat and CLSI standard broth dilution testing (M38-A). We preferred amphotericin $B$ drug because for the life-threatening fungal infections the conventional form of this drug is still the treatment of choice. According to CLSI guideline, for most of the species MIC of amphotericin B are clustered between 0.5 and $2.0 \mu \mathrm{g} / \mathrm{mL}$, MIC above $2 \mu \mathrm{g} / \mathrm{mL}$ have been associated with treatment failures and MIC below $2 \mu \mathrm{g} / \mathrm{Ml}$ with the clinical cure. As per our result, most of the MICs felled in between the MIC range of the CLSI guideline. Only one isolate MIC found to be higher than $2 \mu \mathrm{g} / \mathrm{mL}$. For E- test, no MIC was mentioned by the manufacturer ( $A B$-BIODISK) for amphotericin $B$. So we could not interpret sensitivity/ resistance pattern. E-test MIC was used only for the comparison of E-test MIC and CLSI standard broth dilution testing (M38-A) MIC. In our study, isolates showed good agreement (90\%) within $\pm 2 \log 2$ dilution. Szekely et al. ${ }^{25}$ demonstrated $50 \%$ and $60 \%$ agreement for $\pm 1 \log 1$ and $\pm 2 \log 2$ dilution respectively for amphotericin B. Ingroff et al. ${ }^{26}$ showed $12 \%$ and $31 \%$ agreement for $\pm 1 \log 1$ and $\pm 2 \log 2$ dilution respectively for amphotericin B. This agreement could vary, depending upon the strain.

In general, the discrepancies between $E$ test and broth dilution results were due to higher E test MICs. In our study, the E test Showed comparable results with CLSI M38-A. For antifungal susceptibility testing of mold pathogens E-test has potential value and it is more natural to perform, and less cumbersome compare to M38-A. The wider amphotericin B MICs obtained by the E-test for Aspergillus flavus and Rhizopus arrhizus also suggest that this method could also be useful for MIC detecting.

\section{CONCLUSION}

The prevalence rate of fungal Rhinosinusitis was found to be $22.9 \%$ among the clinically suspected patients of chronic
Rhinosinusitis, and our region may be considered a moderate to higher prevalence area after comparing it with data from the other regions.

Aspergillus flavus was most commonly isolated from patients with fungal Rhinosinusitis establishing it as the commoner etiological agent in our region.

On antifungal susceptibility, 11 out of 12 isolates were found to be susceptible to amphotericin B by CLSI standard broth dilution testing M38-A. So, amphotericin B should be used by the clinician in confirmed cases of FRS. The E-test result was also found to be comparable with M38-A. On comparison of E-test MICs and M38-A MICs, all isolates had shown good agreement at $\pm 2 \log 2$ dilution. However, we found that M38-A is a time-consuming and cumbersome procedure compare to the $\mathrm{E}$ test.

\section{ACKNOWLEDGMENTS}

None.

\section{CONFLICT OF INTEREST}

The authors declare that there is no conflict of interest.

\section{AUTHORS' CONTRIBUTION}

All authors listed have made a substantial, direct and intellectual contribution to the work, and approved it for publication.

\section{FUNDING}

None.

\section{DATA AVAILABILITY}

All datasets generated or analyzed during this study are included in the manuscript

\section{ETHICS STATEMENT}

Prior approval has been taken from the institutional ethical committee.

\section{REFERENCES}

1. Shetty S, Chandrashekar S, Aggarwal N. A Study on the Prevalence and Clinical Features of Fungal Sinusitis in Chronic Rhinosinusitis. Indian J Otolaryngol Head Neck Surg. 2020;72(1):117-122. doi: 10.1007/s12070-01901769-w

2. Lanza DC. Diagnosis of chronic rhinosinusitis. Annals of Otology, Rhinology \& Laryngology. 2004;113:10-14. doi: $10.1177 / 00034894041130 \$ 504$ 
3. Anand VK. Epidemiology and economic impact of rhinosinusitis. Annals of Otology, Rhinology \& Laryngology. 2004;113:3-5. doi: 10.1177/00034894041130\$502

4. Beule A. Epidemiology of chronic rhinosinusitis, selected risk factors, comorbidities, and economic burden. GMS Current Topics in Otorhinolaryngology, Head and Neck Surgery. 2015;14:11.

5. Smith KA, Orlandi RR, Rudmik L. Cost of adult chronic rhinosinusitis: A systematic review. The Laryngoscope. 2015;125(7):1547-1556. doi: 10.1002/lary.25180

6. Chowdhary A, Sharma C, Meis JF. AzoleResistant Aspergillosis: Epidemiology, Molecular Mechanisms, and Treatment. J Infect Dis 2017;216(suppl_3):S436-S444. doi: 10.1093/infdis/ jix210

7. Vipula VA, Amatullah F, Paul S, Lakshmi B. Bacteriological Profile of Chronic Rhino Sinusitis. Int J Curr Microbiol Appl Sci. 2018;7(7):999-1009. doi: 10.20546/ijcmas.2018.707.121

8. Singh V. Fungal Rhinosinusitis: Unravelling the Disease Spectrum. Journal of Maxillofacial and Oral Surgery. 2019;18(2):164-179. doi: 10.1007/s12663-01801182-w

9. Jain S, Das S, Gupta N, Malik JN. Frequency of fungal isolation and antifungal susceptibility pattern of the fungal isolates from nasal polyps of chronic rhinosinusitis patients at a tertiary care centre in north India. Medical Mycology. 2013;51(2):164-169. doi: 10.3109/13693786.2012.694486

10. Alastruey-Izquierdo A, Melhem MS, Bonfietti LX Rodriguez-Tudela JL. Susceptibility test for fungi: Clinical and laboratorial correlations in medical mycology. Rev Inst Med Trop Sao Paulo. 2015;57(Suppl 19):57-64. doi: 10.1590/S0036-46652015000700011

11. Aghakhani A, Eslamifar A, Abyaneh MR, et al. Frequency And Identification Of Fungal Strains In Patients With Chronic Rhinosinusitis. Iranian Journal of Pathology. 2008;3(3):135-149.

12. Razmpa E, Khajavi M, Hadipour-Jahromi M, Kordbacheh $P$. The prevalence of fungal infections in nasal polyposis. Acta Med Iranlca. 2007;45(1):45-50.

13. Venugopal PV, Venugopal TV, Babakrishnan K, Rathinam T, Ulaganathan M, Purushothaman PK. Chronic fungal sinusitis in Tamilnadu, India. Journal de Mycologie Medicale. 2008;18(4):216-223. doi: 10.1016/j.mycmed.2008.09.004

14. Chakrabarti A, Sharma SC, Chandler J. Epidemiology and pathogenesis of paranasal sinus mycoses. Otolaryngol Head Neck Surg. 1992;107(6 Pt 1):745750. doi: $10.1177 / 019459988910700606.1$
15. Jahromi SB, Khaksar AA. Paranasal sinus mycosis in suspected fungal sinusitis. Infectious Diseases and Tropical Medicine Research Center. 2006;1(1):25-29.

16. Singh N, Bhalodiya NH. Allergic fungal sinusitis (AFS)--earlier diagnosis and management. $J$ Laryngol Otol. 2005;119(11):875-881. doi: 10.1258/002221505774783412

17. Dall'Igna C, Palombini BC, Anselmi F, Araujo E, Dall'Igna DP. Fungal rhinosinusitis in patients with chronic sinusal disease. Braz J Otorhinolaryngol. 2005;71(6):712-720. doi: 10.1016/S1808-8694(15)31237-4

18. Joshi RR, Bhandary S, Khanal B, Singh RK. Fungal maxillary sinusitis: a prospective study in a tertiary care hospital of eastern Nepal. Kathmandu University Medical Journal (KUMJ). 2007;5(2):195-198.

19. Goh BS, Gendeh BS, Rose IM, Pit S, Samad SA. Prevalence of allergic fungal sinusitis in refractory chronic rhinosinusitis in adult Malaysians. Otolaryngol Head Neck Surg. 2005;133(1):27-31. doi: 10.1016/j. otohns.2005.03.028

20. Braun $\mathrm{H}$, Buzina $\mathrm{W}$, Freudenschuss $\mathrm{K}$, Beham A, Stammberger $\mathrm{H}$. 'Eosinophilic fungal rhinosinusitis': a common disorder in Europe? The Laryngoscope. 2003;113(2):264-269. doi: 10.1097/00005537200302000-00013

21. Surya Prakash Rao G, Mann SB, Talwar P, Arora MM. Primary mycotic infection of paranasal sinuses. Mycopathologia. 1984;84(2-3):73-76. doi: 10.1007/ BF00436515

22. Panda NK, Sharma SC, Chakrabarti A, Mann SB. Paranasal sinus mycoses in north India. Mycoses. 1998;41(7-8):281-286. doi: 10.1111/j.1439-0507.1998. tb00339.x

23. Alrajhi AA, Enani M, Mahasin Z, Al-Omran K. Chronic invasive aspergillosis of the paranasal sinuses in immunocompetent hosts from Saudi Arabia. The Am J Trop Med Hyg. 2001;65(1):83-86. doi: 10.4269/ ajtmh.2001.65.83

24. Iwen PC, Rupp ME, Hinrichs SH. Invasive mold sinusitis: 17 cases in immunocompromised patients and review of the literature. Clin Infect Dis. 1997;24(6):1178-1184. doi: 10.1086/513662

25. Szekely A, Johnson EM, Warnock DW. Comparison of E-test and broth microdilution methods for antifungal drug susceptibility testing of molds. J clin Microbiol. 1999;37(5):1480-1483. doi: 10.1128/JCM.37.5.14801483.1999

26. Espinel-Ingroff A. Comparison of the E-test with the NCCLS M38-P method for antifungal susceptibility testing of common and emerging pathogenic filamentous fungi. J Clin Microbiol. 2001;39(4):13601367. doi: 10.1128/JCM.39.4.1360-1367.2001 